\title{
A native fungal symbiont facilitates the prevalence and development of an invasive pathogen-native vector symbiosis
}

\author{
Lilin Zhao, ${ }^{1}$ Min Lu, ${ }^{1,2}$ Hongtao Niu, ${ }^{3}$ Guofei Fang, ${ }^{4}$ Shuai Zhang, ${ }^{1}$ and Jianghua Sun ${ }^{1,5}$ \\ ${ }^{1}$ State Key Laboratory of Integrated Management of Pest Insects and Rodents, Institute of Zoology, Chinese Academy of Sciences, \\ Beijing 100101 China \\ ${ }^{2}$ Tree Protection Co-operation Programme, Forestry and Agricultural Biotechnology Institute, University of Pretoria, \\ Pretoria 0002 South Africa \\ ${ }^{3}$ Institute of Plant Protection, Jiangsu Academy of Agricultural Sciences, Nanjing 210014 China \\ ${ }^{4}$ General Station of Forest Pest and Diseases Control, State Forestry Administration, Shenyang 110034 China
}

\begin{abstract}
Invasive pathogen-insect symbioses have been extensively studied in many different ecological niches. Whether the damage of symbioses in different introduced regions might be influenced by other microorganisms has, however, received little attention. Eight years of field data showed that the varied levels of the nematode and beetle populations and infested trees of the invasive Bursaphelenchus xylophilus-Monochamus alternatus symbiosis were correlated with patterns in the isolation frequencies of ophiostomatoid fungi at six sites, while the laboratory experiments showed that the nematode produced greater numbers of offspring with a female-biased sex ratio and developed faster in the presence of one native symbiotic ophiostomatoid fungus, Sporothrix sp. 1. Diacetone alcohol (DAA) from xylem inoculated with Sporothrix sp. 1 induced B. xylophilus to produce greater numbers of offspring. Its presence also significantly increased the growth and survival rate of $M$. alternatus, and possibly explains the prevalence of the nematode-vector symbiosis when Sporothrix sp. 1 was dominant in the fungal communities. Studying the means by which multispecies interactions contributed to biogeographical dynamics allowed us to better understand the varied levels of damage caused by biological invasion across the invaded range.

Key words: Bursaphelenchus xylophilus; Eastern China; infection vector; invasion; Monochamus alternatus; multispecies interactions; ophiostomatoid fungi; pathogen-insect symbioses.
\end{abstract}

\section{INTRODUCTION}

Pathogen-insect symbioses commonly play important roles in the invasion and successful spread of alien species into new environments (von Broembsen 1989, McLeod et al. 2005, Lu et al. 2010, Himler et al. 2011). The symbiotic partners (e.g., fungi, nematodes, bacteria, or viruses) can facilitate the establishment and population increase of their insect vectors in nonnative habitats (Jiu et al. 2007, Himler et al. 2011, Lu et al. 2011). In turn, the vector insects are fundamentally important in the dispersal of introduced plant pathogens such as fungi, viruses, and nematodes (Johannesen et al. 2008, Maehara 2008). However, the unanswered or often overlooked question is whether other native microorganisms might influence the development and damage caused by invasive pathogen-insect symbioses in different introduced natural ecosystems.

The relationship of an invasive plant parasitic nematode, its native vector beetle, and microbial partners provides an ideal opportunity to study the multispecies interactions and invasiveness. The plant

Manuscript received 21 December 2012; revised 10 April 2013; accepted 23 May 2013. Corresponding Editor: D. M. Rizzo.

${ }^{5}$ Corresponding author. E-mail: sunjh@ioz.ac.cn pathogenic pinewood nematode Bursaphelenchus $x y$ lophilus (Nematoda: Aphelenchida: Parasitaphelenchidae) is of little importance in its native range in North America (Wingfield et al. 1984). In contrast, the nematode has caused extensive damage as an invasive alien in Japan, South Korea, China (Mamiya 1983, Yang et al. 2003, Togashi and Jikumaru 2007), and recently Portugal and Spain (EPPO/OEPP 2009). The disease has devastated more than one million hectares of pine forests and, specifically in China, has killed 44 million pine trees between 1982 and 2007 (Yu et al. 2011) and resulted in losses of more than US $\$ 20$ billion (State Forestry Administration of the People's Republic of China 2011).

In infested forests, the nematode is found in the host pine trees together with many other species including the vector beetle Monochamus spp. (Insecta: Coleoptera, Cerambycidae) and ophiostomatoid fungi (Maehara 2008, Akbulut and Stamps 2012). After it was introduced into Asia, B. xylophilus formed a symbiotic relationship with native M. alternatus (Linit 1988, Kanzaki and Futai 2001). The beetle is the only known agent responsible for transmitting the nematode to the host pine trees in the natural forest systems in Asia (Yang et al. 2003, Maehara 2008, Akbulut and Stamps 2012; also see Fig. 1). The female M. alternatus then 


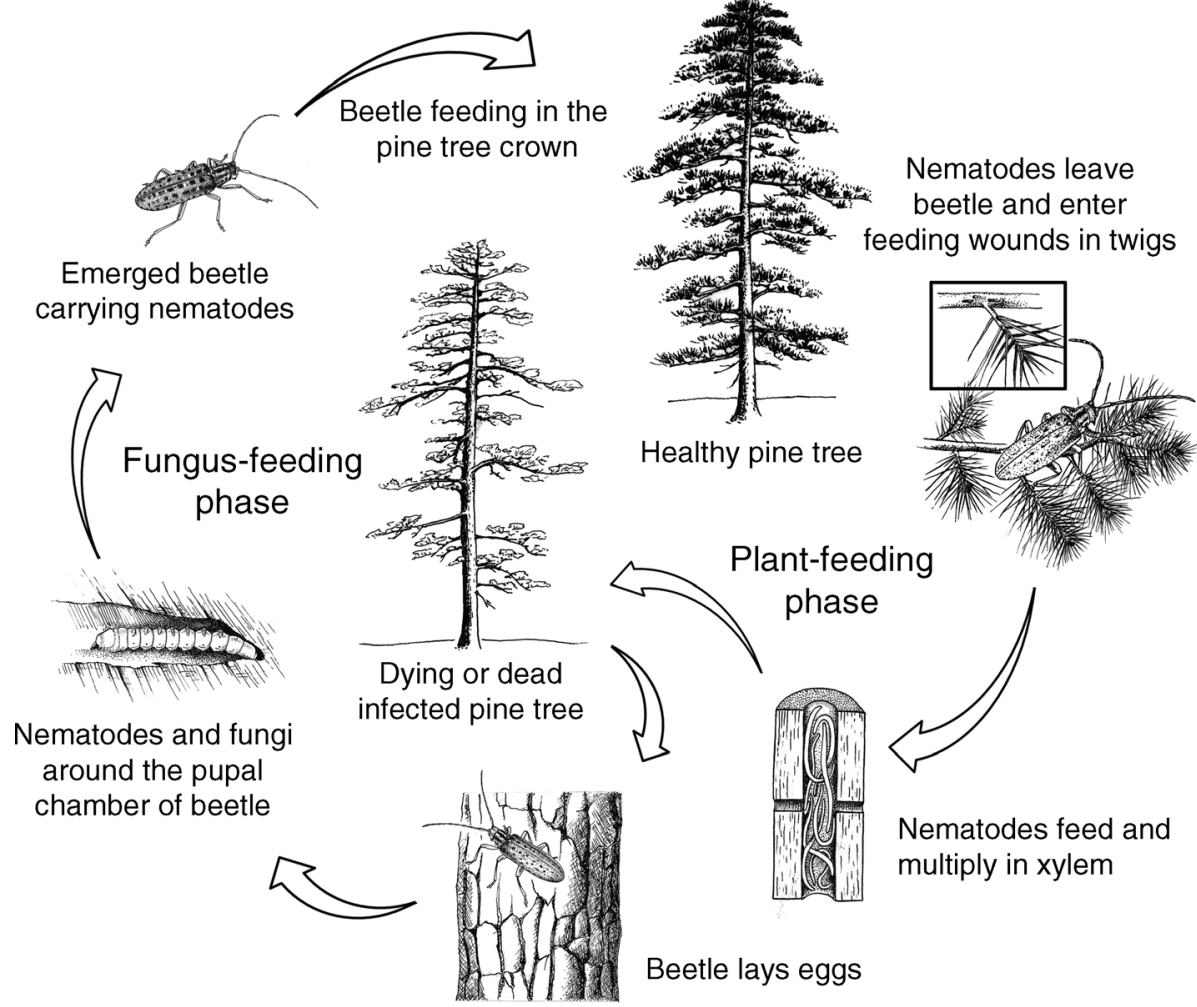

FIG. 1. Relationships among the pinewood nematode (Bursaphelenchus xylophilus), beetle (Monochamus alternatus), and ophiostomatoid fungi in forests in Eastern China.

utilized the nematode-infected and dying pine trees for oviposition (Yang et al. 2003). In this way, the nematode-beetle symbiosis caused the death of large numbers of pine trees. Importantly, these dying pine trees were infested with bark beetles (Coleoptera: Scolytinae) that carry a wide range of ophiostomatoid (blue stain) fungi (Donald et al. 2003). It is these fungi, including Ophiostoma ips, O. minus, and Ceratocystis sp., reported from wood infested with the nematode and vector beetles in Japan, Korea, and America, that provide food for the mycophagous phase of the nematode life cycle (Kobayashi et al. 1974, Wingfield 1987, Hyun et al. 2007).

Ophiostomatoid fungi identified in dead trees positively influence reproduction of the pinewood nematode and the interactions of nematode and host pine trees (Maehara 2008, Maehara and Futai 2000, Niu et al. 2012). This is especially true for native Ophiostoma minus, which was isolated in Japan and was shown to strongly and positively influence the reproduction of the nematode and consequently the number of nematodes dispersed by beetles (Maehara and Futai 2000). Although it is assumed that the ophiostomatoid fungi play a similar role in the life cycle of the pinewood nematode in China, this has not been empirically tested.

Similar symbiotic associations have been extensively reported among microbial associates and wood-boring beetles (Cardoza et al. 2006, Adams et al. 2011, Six et al.
2011, Sun et al. 2013). For example, mycangial symbiotic fungi enhance the diet of Dendroctonus frontalis larvae by contributing to the acquisition of $\mathrm{N}$ and P (Ayres et al. 2000); the yeasts isolated from Phoracantha semipunctata feeding on Eucalyptus could degrade saccharose, maltose, amidon, and pectin (Dowd 1992). Additionally, the complex interactions among host pine and a symbiotic fungi vectored by an invasive bark beetle were driven by a host chemical compound 3carene (Lu et al. 2010, Sun et al. 2013). However, the multispecies interactions between the nematode, sawyer beetle, and fungi have not been considered as a possible driving force that might influence the invasion and damage caused by the nematode. Studying these multispecies interactions is of great relevance, as it might offer a more comprehensive understanding of the mechanisms of successful nematode invasion and possible biogeographical distribution.

In this study, laboratory and field investigations were used to test: (1) whether ophiostomatoid fungi were correlated with differential levels of damage by the nematode-beetle symbiosis by investigating the number of infested trees, population densities of nematodebeetle symbiosis, and species of ophiostomatoid fungi at six sites; (2) how ophiostomatoid fungi affect nematodebeetle symbiosis, including the fecundity of the nematode and the growth of the beetle; and (3) whether a chemical signal released by ophiostomatoid fungi affects 
life history traits of the nematode and beetle. Exploration of these multispecies interactions provides a useful approach in understanding potential factors driving the invasion by an alien invasive pathogen. A more detailed understanding of the biological interactions involved will facilitate the prediction and management of these invasive events, which are not only wreaking havoc in global forests, but are also financially devastating. Given the rapid spread and the severe damage caused by the pinewood nematode, a deeper understanding of the multispecies interactions between the nematode-beetle and ophiostomatoid fungi is justified.

\section{Materials and Methods}

Relationship between damage by the nematode-beetle symbiosis and ophiostomatoid fungi in the field

Damage by the nematode-beetle symbiosis.-Annual damage caused by the nematode-beetle symbiosis from 2004 to 2011 was analyzed and compared for six localities (Appendix: Table A1).The area investigated at each forest site was $\sim 50-60 \mathrm{~km}^{2}$. The site characteristics are shown in the Appendix, Table A1. The number of newly dead trees resulting from infestation by the nematode and beetle was enumerated annually for each site, each October. Trees were identified as infested by nematode-beetle symbiosis based upon the browning of the needles, reduced resin flow, presence of beetle oviposition sites in the phloem, and larval feeding in the xylem. Nematodes were extracted from the infested trees and identified microscopically (Zhao et al. 2007).

Population density of the nematode.-To estimate the relationship of the base population of nematodes that would be carried by the vector beetle in April and the corresponding damage of the nematode-beetle symbiosis in October of the same year, we investigated the number of nematodes in the wood around the beetle pupal chambers each April from 2004 to 2011. At each site, three infested dead trees with a dbh (diameter at breast height) of $10-15 \mathrm{~cm}$ were chosen for study. One pupal chamber was randomly sampled from the southern side of the xylem in a chosen tree at breast height for each $10-20 \mathrm{~km}^{2}$. About $20 \mathrm{~g}$ of wood around each pupal chamber were sampled and placed in Baermann funnels to recover nematodes, which were then counted by direct observation through a dissecting microscope. The average number of nematodes around pupal chambers from each site was used to estimate the density of the nematode each April.

Population density of the beetle.-To estimate the relationship of the base population of beetles from host pine trees in April and the subsequent damage from the nematode-beetle symbiosis in October of the same year, at each site, three newly dead trees infested by the nematode-beetle symbiosis with a dbh of $10-15 \mathrm{~cm}$ were chosen for study each April from 2004 to 2011. The number of beetles in the trunk (3-4 m long, 5-10 cm diameter) of the chosen tree was investigated for each $10-20 \mathrm{~km}^{2}$. Average numbers of beetles per meter of dead tree trunk $(5-10 \mathrm{~cm}$ diameter) were counted to determine the beetle density for each site.

Body mass of the beetle and the number of nematodes carried by the beetle vector.-To investigate whether the adult body size affects the number of nematodes carried by individual beetles, nematodes were extracted using Baermann funnels from 30 adult beetles (which had been weighed) in the Forestry Center of Chashan. Nematodes were then counted by direct observation using a dissecting microscope in June 2007.

Ophiostomatoid fungi.-Field surveys were conducted to determine which ophiostomatoid fungi were associated with the beetle and nematode in the beetles' pupal chambers at the six selected sites. At each site, 30 dead infested trees with a dbh of $10-15 \mathrm{~cm}$ were chosen (one tree per 1-2 $\mathrm{km}^{2}$ ) for study, and one pupal chamber was sampled at breast height from the xylem on the south side of the chosen tree in April 2007. Thirty samples were taken from each site, 180 samples for the six sites. Nematode presence in the samples was determined using the Baermann funnel technique (Zhao et al. 2007).

All samples were maintained separately and frozen at $-70^{\circ} \mathrm{C}$ for 20 minutes to kill nematodes before fungi were isolated. Each pupal chamber was cut into 3-5 pieces (each about $4 \mathrm{~cm}^{2}$ ) using a dissection knife. Wood samples from each chamber were plated onto agar selective for ophiostomatoid fungi, as described by Lu et al. (2009). Representative cultures used in this study were deposited in the culture collection (CMW) of the Forestry and Agricultural Biotechnology Institute (FABI), University of Pretoria, Pretoria, South Africa.

Pure cultures of the isolated fungi were sorted into groups based on culture morphology. Single hyphal tip cultures were prepared from cultures representing all morphological groups for DNA sequencing. Cultures were grown in $50 \mathrm{~mL}$ of malt extract broth $(30 \mathrm{~g}$ malt extract with $1 \mathrm{~L}$ distilled water) at $25^{\circ} \mathrm{C}$ in the dark for 10 days. Mycelium was harvested by filtration through Whatman No. 1 filter paper (Whatman Incorporated, Florham Park, New Jersey, USA), freeze-dried, then ground into a fine powder, and DNA was extracted using PrepMan Ultra Sample reagent (Applied Biosystems, Carlsbad, California, USA) following the manufacturer's protocols. PCR and DNA sequencing reactions were prepared and performed as described by Lu et al. (2009). Data sets were compiled in MEGA 4 (Tamura et al. 2007). Sequences were deposited in GenBank under the accession numbers provided in Appendix: Table A2. NCBI similarity scores were obtained using a BLAST search of sequences in GenBank. Fungal species were considered to be a match at the $99 \%$ similarity level.

\section{Effects of ophiostomatoid fungi on populations of nematodes and beetles}

The effect of fungi on population growth of the nematode.-Forty xylem wood blocks (5 cm long, 2.5 $\mathrm{cm}$ diameter) of healthy Pinus massoniana were used for 
bioassays of nematode population growth, as explained by Zhao et al. (2007). The blocks were inoculated with the three ophiostomatoid fungi most commonly isolated in this project, including Ophiostoma ips, Sporothrix sp. 1, and Sporothrix sp. 2. Two weeks later, 1000 nematodes from Fuyang, Zhejiang province in China were inoculated into each block and the wood blocks were maintained at $25^{\circ} \mathrm{C}$ in the dark for 15 days. The nematodes were then extracted from the wood blocks using Baermann funnels.

Reproduction and age structure of the nematode were further tested by inoculating five pairs of nematode adults onto petri dishes (35 $\mathrm{mm}$ diameter) containing each of the three fungi, which were cultivated on $2 \%$ (mass/volume) malt extract agar (MEA) (20 g malt extract, $20 \mathrm{~g}$ agar, and $1 \mathrm{~L}$ deionized water) ( $\mathrm{Lu}$ et al. 2009) at $25^{\circ} \mathrm{C}$ in the dark for seven days. Seventy plates per fungus were used. The living nematodes were separated from 10 randomly selected plates using Baermann funnels every 24 hours and the numbers of juvenile, female, and male nematodes on each fungal plate were enumerated by direct observation through a dissecting microscope. We assumed that the offspring had completed one generation when there were more than five females or males present in a plate. The number of days to complete a generation was estimated from these counts. The total numbers of nematodes on the seventh day were used to estimate the nematode population. The ratio of females to males and the percentage of juveniles on the seventh day were used to estimate the age structure of the nematodes on the three different fungi.

Effects of fungi on larval growth and survival of the beetle.-As adult body size in insects is dependent on the amount of growth that occurs during the larval stage (Tennessen and Thummel 2011), the beetle larval growth and survival were investigated on the isolated fungi. An artificial diet described by Wang et al. (2012) including $400 \mathrm{~mL}$ of distilled water, $15 \mathrm{~g}$ of finely ground $P$. massoniana xylem tissue, and $15 \mathrm{~g}$ of agar was used to test the effects of the fungi on beetle larval growth. The artificial diet $(15 \mathrm{~mL})$ was poured into petri dishes $(75$ $\mathrm{mm}$ diameter), and an isolate of each of the test fungi was inoculated on the surface and cultures (21 replicates for each treatment) were incubated at $25^{\circ} \mathrm{C}$ in darkness for $10 \mathrm{~d}$. Twenty-one beetle larvae were then transferred to the fungus-colonized artificial diets for each of the three fungi. These larvae, weighing between $0.31-0.39 \mathrm{~g}$, were allowed to feed on the fungus-colonized medium at $25^{\circ} \mathrm{C}$ in darkness. Control treatments consisted of 21 dishes containing the artificial diet without fungi. After seven days, larvae that had fed on each of the fungusinfected or control plates were weighed. The average growth of the larvae on each of the three different fungi and the controls was calculated using the following formula: beetle growth rate (change in mass [\%]) = $\left(M_{t}-M_{0}\right) / M_{0} \times 100$, where $M_{t}$ is the mass of the vector larvae treated after feeding for seven days, and $M_{0}$ is the initial mass of the vector larvae before treatment. The 21 larvae on each of the artificial diets, with three containing fungi, were then observed continuously until they molted into pupae, adults, or died, and the survival rates were calculated.

\section{Effects of chemicals extracted from fungus-colonized xylem on nematode and beetle populations}

Chemical analyses.- To analyze the chemical constituents of the fungus-infested wood, xylem wood blocks were inoculated with the three different ophiostomatoid fungi and incubated for 15 days as described above. The xylem constituents were determined using methods described previously (Lu et al. 2010) by taking sections of the xylem stained by the fungus $(0.15 \mathrm{~g})$, chopping these finely with a razor blade, and extracting them in 4 $\mathrm{mL}$ of ethanol for 30 minutes. The extracts were concentrated to $100 \mu \mathrm{L}$, and $2 \mu \mathrm{L}$ of each extract was analyzed by gas chromatograph-mass spectrometer (GC-MS) (Hewlett Packard 6890N GC model coupled with 5973 MSD (Palo Alto, California, USA) equipped with a DB-WAX column $(60 \mathrm{~m}$ length $\times 0.25 \mathrm{~mm}$ ID $\times$ $0.25 \mathrm{~m}$ film) (J and W Scientific, Folsom, California, USA). The GC oven temperature program was set at $50^{\circ} \mathrm{C}$ for one minute, increased to $220^{\circ} \mathrm{C}$ at $5^{\circ} \mathrm{C}$ per minute, and then held at $220^{\circ} \mathrm{C}$ for 10 minutes. The oncolumn injector temperature was $220^{\circ} \mathrm{C}$, and helium was used as the carrier gas (flow rate $=1 \mathrm{~mL}$ per minute). Identifications of chromatogram peaks were based on comparisons with retention times and mass spectra of authentic standards and those in the NIST02 library (Scientific Instrument Services, Ringoes, New Jersey, USA).

The chemical components of the extracts in each xylem sample inoculated with isolated fungi were compared in order to determine functional chemicals from the xylem tissue inoculated with Sporothrix sp. 1 that differed from those identified from Sporothrix sp. 2 and $O$. ips, to elucidate how Sporothrix sp. 1 promoted the propagation of the nematode and beetle growth in the pine tree. The results were quantified using an internal standard (cis-9-hexadecenal) added to each sample. The unique chemical concentrations were also determined on a dry mass of phloem basis by oven drying (following extraction) and weighing each phloem sample bearing fungal lesions.

Effect of diacetone alcohol ( $D A A$ ) on nematode fecundity.-In order to assess the potential effects of diacetone alcohol (DAA), a unique compound found in xylem inoculated with Sporothrix sp. 1, we tested the effects of diacetone alcohol (DAA, ACROS, >99\%) on the reproduction of the nematode. Forty pairs of nematodes (40 females and 40 males) were inoculated onto petri dishes $(90 \mathrm{~mm}$ diameter) colonized by an isolate of the grey mold fungus Botrytis cinerea (commonly used to culture B. xylophilus) and incubated in the dark at $25^{\circ} \mathrm{C}$ for seven days. A piece of filter paper with $50 \mu \mathrm{L}$ of a $10-\mu \mathrm{mol} / \mathrm{L}$ concentration of DAA, 
consistent with the concentrations that are released by $15 \mathrm{~g}$ of xylem wood samples, was placed on the inner sides of the lids of petri dishes. The 10 replicate plates were sealed and incubated at $25^{\circ} \mathrm{C}$ in darkness for seven days. Nematodes were then extracted from individual plates of the cultures using a Baermann funnel and enumerated using a dissecting microscope. For the control treatment, a similar amount of ethanol was added to the inner sides of the lids of petri dishes.

Effect of DAA on the beetle growth.-The xylem agar medium without fungi described previously was supplemented with $50 \mu \mathrm{L}$ DAA $(10 \mu \mathrm{mol} / \mathrm{L})$, similar to the concentrations that are released by $15 \mathrm{~g}$ of xylem wood. For the control treatment, a similar amount of ethanol was added to the autoclaved phloem-agar medium.

Twelve beetle larvae were transferred to the artificial diets for the treatment (12 dishes). After seven days, larvae on each of the fungus-infected and control plates were weighed. The average growth of the larvae was calculated using the following formula: increased rate of beetle's body mass $(\%)=\left(M_{t}-M_{0}\right) / M_{0} \times 100$, where $M_{t}$ is the mass of the vector larvae treated for seven days and $M_{0}$ is the mass of the vector larvae before treatment.

\section{Statistical analyses}

The differences in the number of infested trees in different invaded sites were compared by Kruskal-Wallis nonparametric test, followed by evaluation with the Mann-Whitney $U$ test with Bonferroni correction for multiple comparisons $(\alpha=0.05 / 15=0.0033)$. A chisquare test analysis was used to assess the frequency for Sporothrix sp. 1 from isolated fungal strains in pupal chambers from different invaded sites and survival rates of beetle larvae in different fungi. The differences of the life history traits of $B$. xylophilus and $M$. alternatus on different ophiostomatoid fungi and DAA were compared by one-way ANOVA with Bonferroni correction for multiple comparisons with the following adjusted $\alpha$ levels: $\alpha=0.05 / 6=0.0083$ for the life history traits of the nematode and the beetle in the wood blocks; $\alpha=0.05 / 3=$ 0.017 for comparison of life history traits of the nematode on petri dishes; and $\alpha=0.05 / 1=0.05$ for the effects of DAA on the nematode and beetle. The correlations between the density of the nematode population and the corresponding number of newly infested dead trees in the same year, and the correlation of the frequency for Sporothrix sp. 1 from isolated fungal strains in pupal chambers and the corresponding densities of nematode and beetle populations in the same year, were determined using regression analysis. The correlation of the frequency of Sporothrix sp. 1 from isolated fungal strains in pupal chambers and the corresponding newly dead infested trees in the same year, the correlation between the density of the beetle and the corresponding newly infested dead trees in the same year, and the correlation between the mass of the beetle and the corresponding number of nematodes carried, were determined using polynomial models.

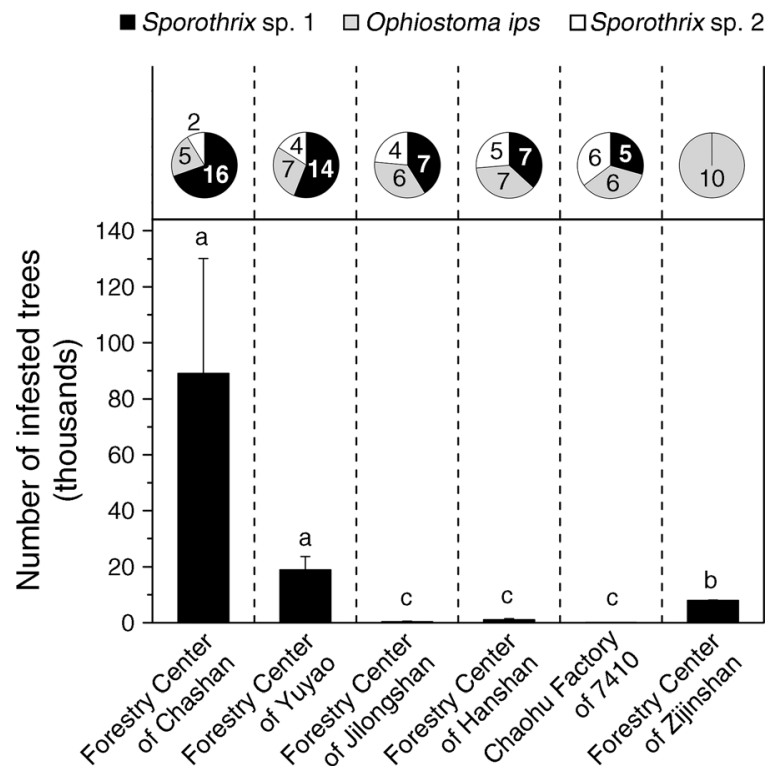

FIG. 2. Ecological damage from the nematode-beetle symbiosis (number of infested Pinus massoniana trees, mean $\pm \mathrm{SE}$ ) and the frequency distribution of ophiostomatoid fungal isolates at six forest sites. The numbers inside the pie diagrams represent the number of isolated fungal strains in beetle pupal chambers from different invaded sites. Bars with different lowercase letters above them differ significantly at $P<0.0033$ (Mann-Whitney $U$ test).

Analyses of data for all experiments were performed using SPSS 11.0 for Windows (SPSS 2002).

\section{Results}

\section{Ecological damage by nematode-beetle symbiosis}

Survey records from 2004 to 2011 showed that the number of newly dead trees infested by the nematodebeetle symbiosis each year at six sites were significantly different (Kruskal-Wallis test, $\chi^{2}=41.46, P=1.55 \times$ $10^{-4}$, df $=5$; Fig. 2). The number of newly dead trees each year was positively correlated with the number of nematodes in the beetle pupal chambers and the population densities of beetles (correlation analysis, $r=$ $0.97, P=1.64 \times 10^{-29}, N=42$ nematodes; $r=0.63, P=$ $5.44 \times 10^{-6}, N=42$ beetles; Appendix: Fig. Ala, b). The number of nematodes carried by the beetles was positively correlated with the mass of beetles (correlation analysis, $r=0.87, P=6.51 \times 10^{-10}, N=30$ ) (Appendix: Fig. A1c).

\section{Ophiostomatoid fungi in different forest areas}

Intense blue staining was commonly found in wood around the beetle pupal chamber walls in all areas of invasion considered in this study (see Plate 1). The frequency of occurrence of blue stain associated with the pupal chambers reached $96.36 \%$.

Three species of ophiostomatoid fungi were identified from the pupal chambers (see Plate 1). These included two undescribed Sporothrix species (Sporothrix sp. 1 and 

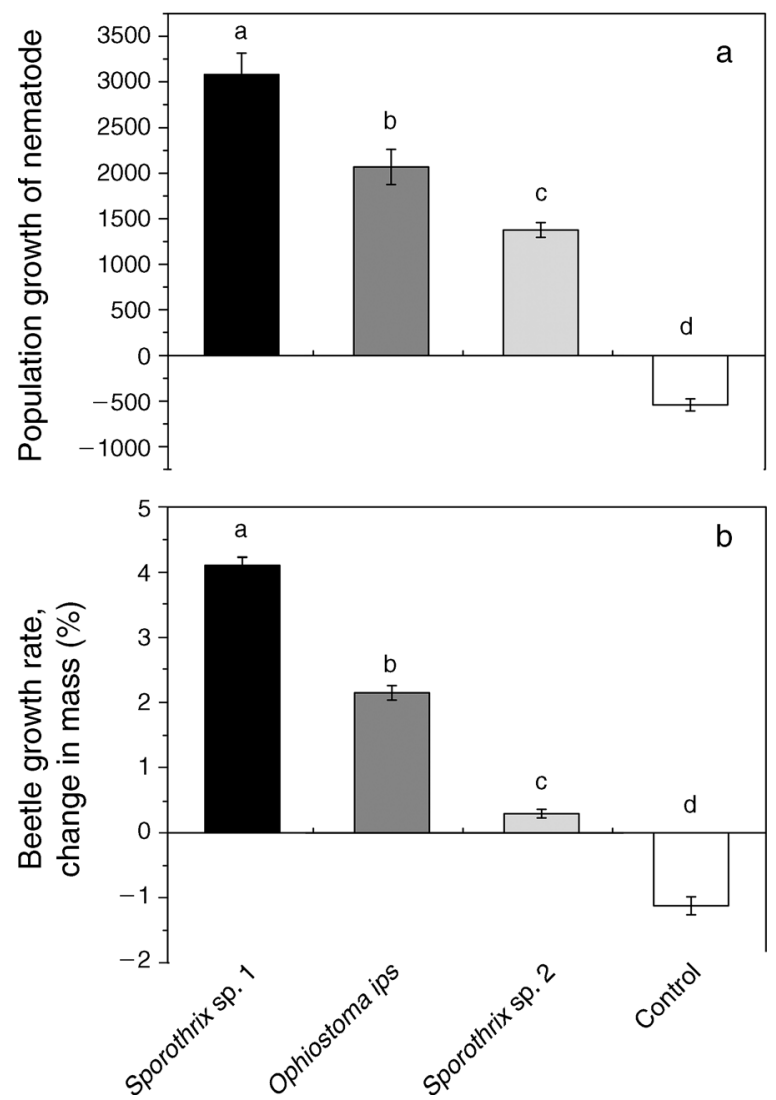

FIG. 3. Effects of three ophiostomatoid fungi (a) on the population increase of the nematode Bursaphelenchus xylophilus and (b) on the body mass of beetle (Monochamus alternatus) larvae over seven days. Error bars indicate SE. Bars with different lowercase letters above them differ significantly at $P<$ 0.0083 (one-way ANOVA).

Sporothrix sp. 2), and a third group of isolates identified as those of Ophiostoma ips (Appendix: Table A2). A total of 111 fungal isolates were obtained from beetle pupal chambers where nematodes were also present (Fig. 2). Of these, Sporothrix sp. 1 was the most abundant fungus found in this study (44.14\%). O. ips and Sporothrix sp. 2 were found in $36.93 \%$ and $18.92 \%$ of isolated fungal strains in pupal chambers, respectively (Fig. 2).

The proportion of Sporothrix sp. 1 isolated relative to the other two fungi differed significantly among six sites (chi-square test, $\chi^{2}=17.33, P=0.004$, df $=5$; Fig. 2). The isolation frequency of Sporothrix sp. 1 comprised $69.57 \%$ and $56 \%$ at the Forestry Center of Chashan and the Forestry Center of Yuyao, respectively, which were greater than the isolation frequencies from other locations (41.18\%, 36.94\%, and 29.41\%, 0\%; Fig. 2). The frequency of Sporothrix sp. 1 relative to the total number of fungal isolates was positively correlated with the overall damage caused by the nematode-beetle symbiosis, the number of nematodes in the beetle pupal chambers, and the population densities of beetles (correlation analysis, $r=0.94, P=0.019, N=5$ for infested trees; $r=0.95, P=0.016, N=5$ for the number of nematodes; $r=0.91, P=0.032, N=5$ for the beetle; Appendix: Fig. A2a-c).

\section{Effects of ophiostomatoid fungi on the population of nematodes and beetles}

The artificially constructed pupal chambers inoculated with Sporothrix sp. 1, Sporothrix sp. 2, or O. ips provided effective food sources for the nematode. The number of nematodes produced on Sporothrix sp. 1 cultures after 10 days was significantly higher than those feeding on $O$. ips or Sporothrix sp. 2 cultures (one-way ANOVA, $F_{3,36}=90.91, P=7.32 \times 10^{-17}, n=10$; Fig. 3a).

The effects of associated ophiostomatoid fungi species on the reproductive traits of nematodes were further tested in petri dishes (90 $\mathrm{mm}$ diameter). Compared with O. ips and Sporothrix sp. 2, the nematodes that fed upon Sporothrix sp. 1 reproduced most actively (one-way ANOVA, $F_{2,27}=116.24, P=5.41 \times 10^{-14}, n=10$; Fig. $4 \mathrm{a})$, and the nematodes completed their first life cycle in less than three days (Fig. 4b). O. ips was a less favorable food source; the nematodes completed their first generation in approximately five days. Sporothrix sp. 2 was the least favorable food source; the generation time was approximately six days, more than twice as long as for Sporothrix sp. 1 (one-way ANOVA, $F_{2,27}=51.85, P$ $=5.67 \times 10^{-10}, n=10$; Fig. $\left.4 \mathrm{a}, \mathrm{b}\right)$. The sex ratio and the percentage of juveniles of nematodes that fed upon Sporothrix sp. 1 were also higher than those that fed upon $O$. ips and Sporothrix sp. 2, respectively (one-way ANOVA, $F_{2,27}=63.17, P=6.57 \times 10^{-11}, n=10 ; F_{2,27}=$ 11.04, $P=3.33 \times 10^{-4}, n=10$; Fig. 4c, d).

The artificial diets containing the three fungal strains (Sporothrix sp. 1, 2, and O. ips) were all effective food sources for the beetle larvae. The rate of increase of body mass of larvae that fed on a diet containing Sporothrix sp. 1 for seven days was greater than larvae that fed upon a diet containing either $O$. ips or Sporothrix sp. 2 (one-way ANOVA, $F_{3,80}=404.42, P$ $=3.16 \times 10^{-48}, n=21$; Fig. $\left.3 \mathrm{~b}\right)$. The survival rate of larger larvae on Sporothrix sp. 1 was $90.47 \%$, significantly higher than on Sporothrix sp. 2, O. ips, and control (chi-square test, $71.43 \%, 57.14 \%$, and $42.86 \%$ respectively, $\chi^{2}=11.53, P=0.009$, $\mathrm{df}=3, n=21$ ).

\section{$D A A$ effects of xylem-inoculated Sporothrix sp. 1 on nematodes and beetles}

Diacetone alcohol (DAA) was more abundant in the xylem tissue inoculated by Sporothrix. sp. 1 than on that inoculated with $O$. ips or Sporothrix sp. 2 (Fig. 5a). The presence of $10 \mu \mathrm{mol} / \mathrm{L}$ DAA, a concentration consistent with those released by $15 \mathrm{~g}$ of xylem wood samples, increased the fecundity of the nematodes. The 40 pairs of adult nematodes produced greater numbers of larvae in the petri dishes $(7428 \pm 981.59$ larvae, mean $\pm \mathrm{SE})$ than in the control treatments $(1940 \pm 294.28$ larvae $)$ 
(one-way ANOVA, $\mathrm{F}_{1,18}=43.68, P=3.32 \times 10^{-6}, n=$ 10; Fig. 5b). The growth of beetles was also assessed based on their mass increase after feeding on the diet for seven days. The presence of $10 \mu \mathrm{mol} / \mathrm{L}$ DAA improved the growth of the beetle larvae $(2.28 \% \pm 0.30 \%$; one-way ANOVA, $F_{1,22}=68.32, P=3.42 \times 10^{-8}, n=12$; Fig. 5 c).

\section{DiscusSION}

An extensive field survey of the pinewood nematode, its beetle vector, and ophiostomatoid fungi in China suggested both a prevalence of Sporothrix sp. 1 correlated with higher tree mortality, which positively influenced the populations of this invasive alien nematode and its native vector beetle. Given that it has not been found in previous studies of the fungi associated with the nematode in the United States (Wingfield et al.1984), Sporothrix sp. 1 is most likely a native ophiostomatoid fungus in China. Ophiostomatoid fungi were isolated from the pinewood nematode and its vector in South Korea. However, it is possible that the fungi might be introduced into pine trees by secondary bark beetle colonization of the trees (Donald et al. 2003, Hyun et al. 2007). Here we demonstrate that Sporothrix sp. 1 had a strong positive effect on the population and prevalence of invasive pinewood nematode-native beetle symbiosis in the xylem of trees. Other studies have also shown the positive impact of microbial partners on herbivores in plant invasions (Christian 2001, Shah et al. 2012). However, there have been no studies that demonstrate a tripartite partnership of invasive nematodes, native beetles, and ophiostomatoid fungi interactions that facilitate an invasion.

While it is well recognized that ecological impacts differ substantially in the introduced and native ranges of invasive organisms (von Broembsen 1989, Hulme 2008, Moloney et al. 2009), the different levels of damage of invasive species in different areas of introduction are poorly understood (Dybdahl and Kanke 2005). Although the areas of pinewood nematode invasion considered in this study have similar climates (Pan et al. 2009), there were distinctly different levels of damage over a period of eight years. These results suggest that the differences can be attributed to the population density of the nematode and its vector beetles, which are both influenced positively by the presence of a certain species of fungus.

Previous studies have shown that symbiotic microbes can positively influence the life history traits of an invasive species (Jiu et al. 2007, Himler et al. 2011, Lu et al. 2011). We found a somewhat different result in this study; the fungal partner influenced the development of both the invader nematode and its vector beetle. It was clear that Sporothrix sp. 1 had a positive influence on the life history of both the nematode and beetle. The nematode produced greater numbers of offspring with a female-biased sex ratio, and developed faster in the presence of one native ophiostomatoid fungus, Sporothrix sp. 1. The fragrant DAA released from wood

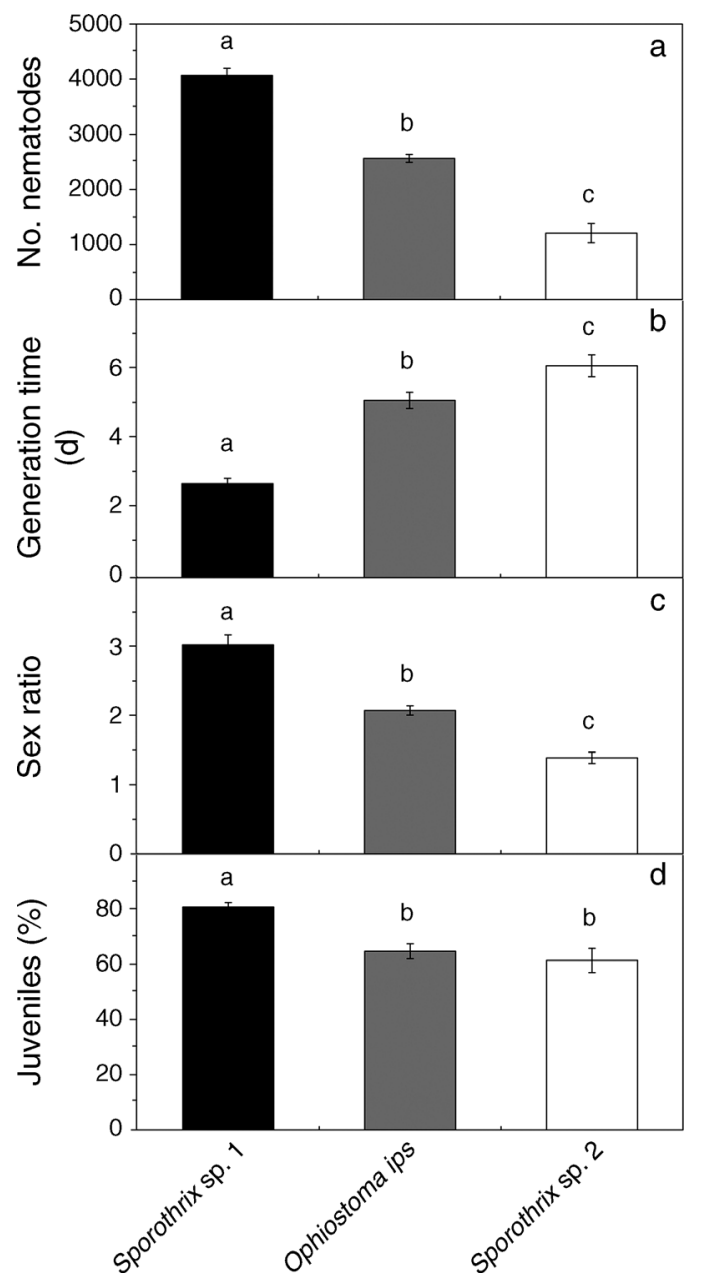

FIG. 4. Effects of associated species of ophiostomatoid fungi on the reproduction traits of the nematode: (a) nematode population size; (b) generation time of the nematode; (c) sex ratio (female: male) of the nematode. (d) percentage of young juvenile nematodes. Values represent the means and standard errors of 10 replicates for each treatment. Bars with different lowercase letters above them differ significantly at $P<0.017$ (one-way ANOVA).

infected by Sporothrix sp. 1 promoted the fecundity of the nematode and both beetle growth and survival, and further facilitated the positive interactions of the pinewood nematode and vector beetles, thus promoting the invasion of the nematode. Although the invasive alien species have already been shown to affect biodiversity and alter ecosystem processing in the introduced ranges (Roques 2012), this leads us to speculate that an invasive alien species could adapt to and utilize the native biological resources available to establish a new and unique multispecies partnership. Such new interactions among organisms in novel environments could increase the intrinsic invasiveness of pests and pathogens, positively influencing their survival, reproduction, and spread. 
a

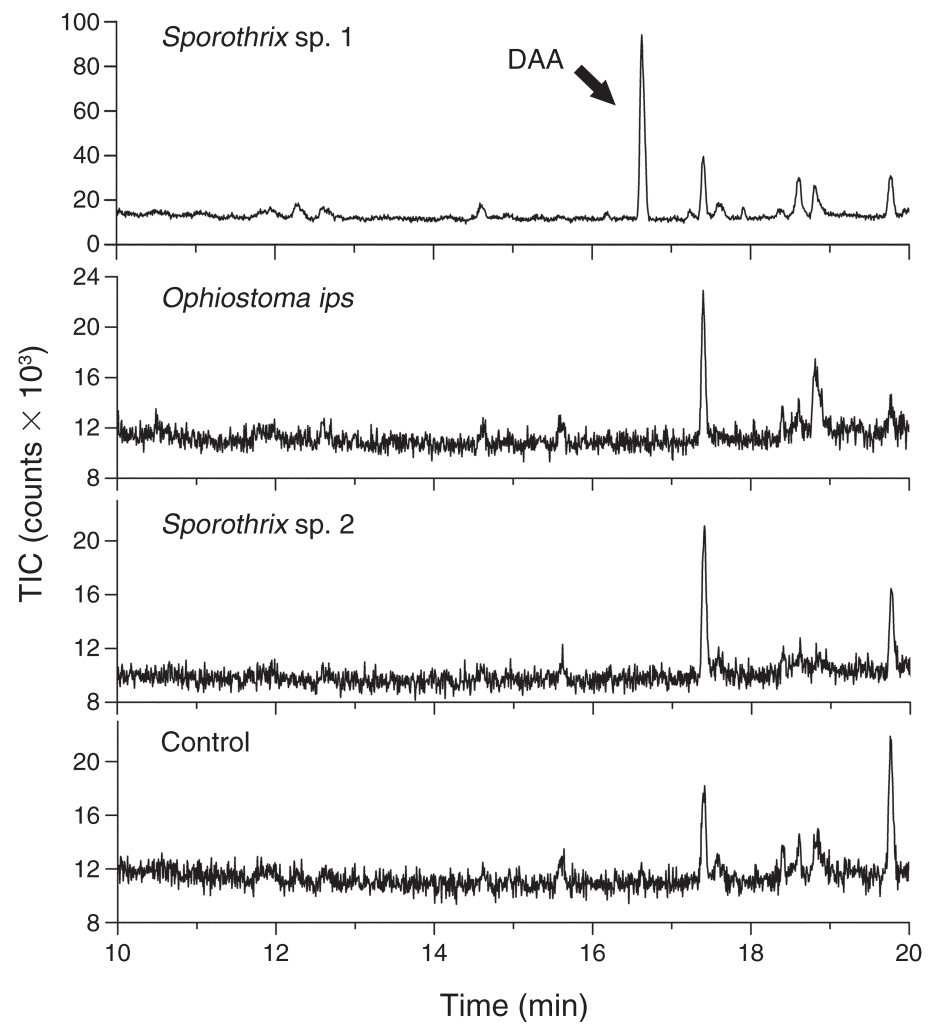

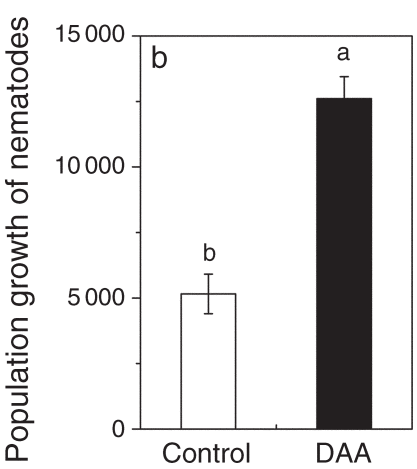

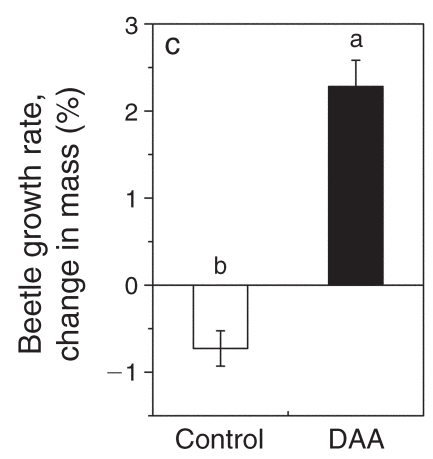

FIG. 5. Effect of diacetone alcohol (DAA) from xylem tissue inoculated with Sporothrix sp. 1 on the populations of the nematode and the growth of the beetle. (a) DAA was found only in the xylem inoculated by Sporothrix sp. 1 (TIC is total ion current). (b) Effect of DAA on the population increase of the nematode. (c) Effect of DAA on the body mass of the beetle. Error bars indicate SE. Bars with different lowercase letters above them differ significantly at $P<0.001$ (one-way ANOVA).

Guimarães et al. (2011) reported on the coevolution of two species in symbiotic networks. The addition of a third partner influencing the symbiosis of an invasive alien species now provides an opportunity to explore the extrinsic drivers of adaptive evolution. The nematode has existed in a novel environment for about 30 years in China (Yang et al. 2003). We speculate that the nematode has evolved an intimate interaction with Sporothrix sp. 1. In order to better understand this situation, it will be necessary to analyze the adaptive evolution of genetic expression related to reproduction, dispersal, and virulence of the nematode in different regions of introduction and on different ophiostomatoid fungi. In this way, it should be possible to consider the variable adaptive responses to the biological factors in these regions.

Elucidation of multispecies interactions between invasive species and native partners could offer novel strategies to forecast and control invasive species. Melbourne and Hastings (2009) showed that the observed variation among invasions cannot be explained by demographic stochasticity alone, which indicates inherent limitations to predictability in the simplest ecological settings. Ebeling et al.(2008) also suggested that data on multispecies interactions could be used to predict the degree of potential invasiveness of an invader, in some cases more effectively than models based simply on life history data. Despite the fact that the Chinese government is investing large amounts of money and effort to forecast and manage pinewood nematode outbreaks, few effective methods have been found (Yu et al. 2011). Based on the results of this study, it might be possible to predict the distribution and population density of the nematode in newly introduced areas by using population models of multispecies interactions. This information could then be used to develop management prescriptions to decrease the spread of the invasive species and to reduce its impact. Overall, this study has shown that different microbial species involved in complex interactions with invasive nematodes and vector beetles could influence the success of the invasion. This has important implications regarding the understanding of basic biological concepts underpinning the assembly of novel communities, as well as the practical management of invasive species.

\section{ACKNOWLEDGMENTS}

We thank Jin Shi, Liya Jiang (Forest Pests Control and Quarantine Bureau of Anhui Province), and Zongbin Tang (Forest Pests Control and Quarantine Bureau of Chaohu) for their assistance in field data collection. We are especially grateful to two anonymous reviewers for their constructive comments, Michael J. Wingfield (Forestry and Agricultural 

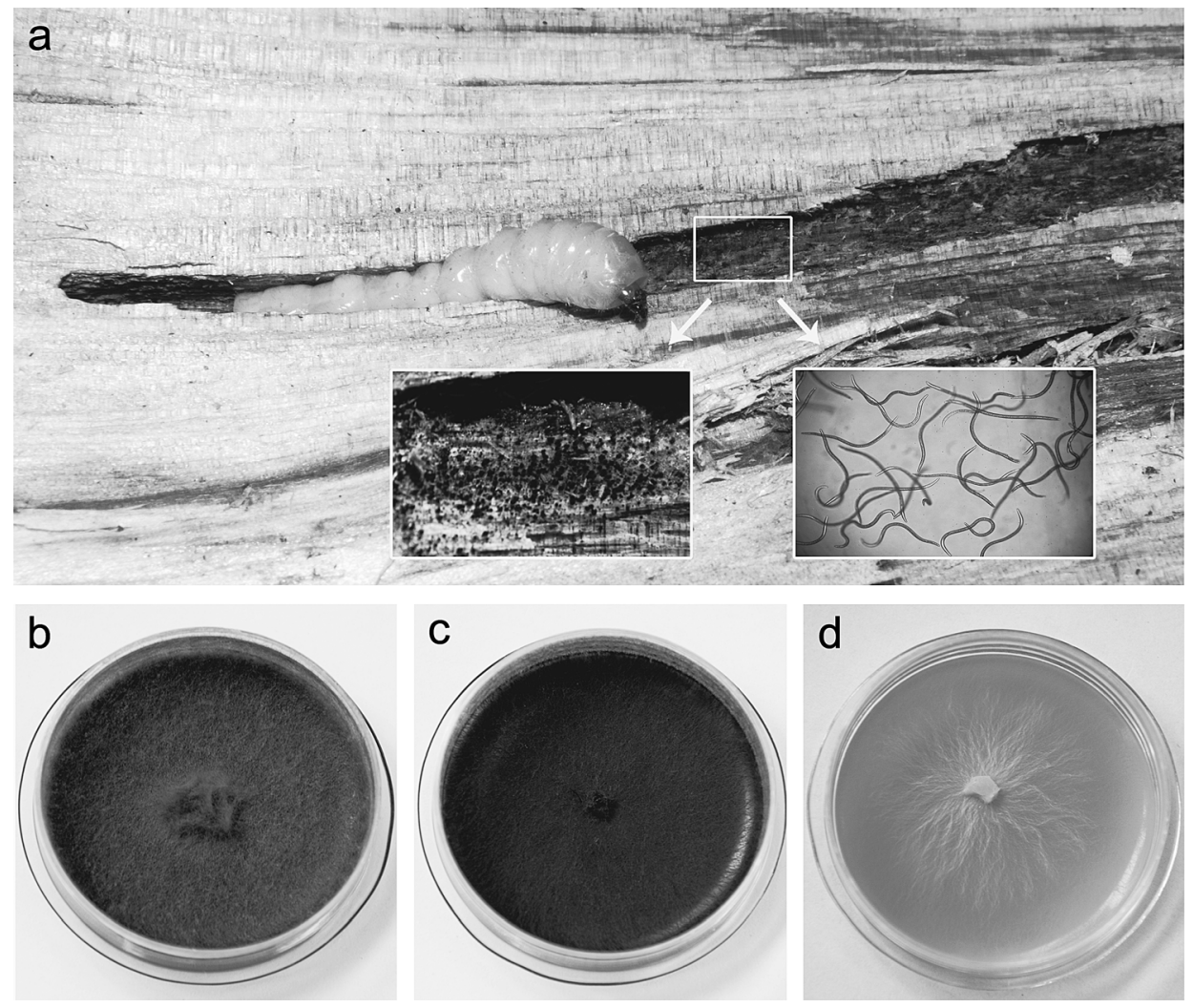

Plate 1. (a) Ophiostomatoid fungi and pinewood nematode found around pupal chambers of the vector beetle in China. (b, c, d) Cultures of Sporothrix sp. 1, Ophiostoma ips, and Sporothrix sp. 2. Photo credits: S. Zhang.

Biotechnology Institute, University of Pretoria, South Africa), Leland Humble (Canadian Forest Service), and Jacob Wickham (Institute of Chemistry, Chinese Academy of Sciences), Gary Man (USDA Forest Service), and Christian Salcedo (Institute of Zoology, CAS) for reviews of earlier versions of the manuscript. Thanks are also extended to Wilhelm Z. Debeer (Forestry and Agricultural Biotechnology Institute, University of Pretoria, South Africa) for identification of fungal strains. This work was supported by the National Basic Science Research Program (2009CB119204 and 2012CB114105), National Natural Science Foundation of China (31272323, 31221091, 31370650, and 30970369), the CAS Knowledge Innovation Key Research Program (KSCX2-EW-J-2), and the European Seventh Framework Project REPHRAME. Authors L. Zhao and M. Lu contributed equally to this work.

\section{Literature Cited}

Adams, A. S., C. K. Boone, J. Bohlmann, and K. F. Raffa. 2011. Responses of bark beetle-associated bacteria to host monoterpenes and their relationship to insect life histories. Journal of Chemical Ecology. http://dx.doi.org/10.1007/ s10886-011-9992-6

Akbulut, S., and W. T. Stamps. 2012. Insect vectors of the pinewood nematode: a review of the biology and ecology of Monochamus species. Forest Pathology 42:89-99.

Ayres, M. P., R. T. Wilkens, J. J. Ruel, M. J. Lombardero, and E. Vallery. 2000. Nitrogen budgets of phloem-feeding bark beetles with and without symbiotic fungi. Ecology 81:21982210.

Cardoza, Y. J., K. D. Klepzig, and K. F. Raffa. 2006. Bacteria in oral secretions of an endophytic insect inhibit antagonistic fungi. Ecological Entomology 31:636-645.
Christian, C. E. 2001. Consequences of a biological invasion reveal the importance of mutualism for plant communities. Nature 413:635-639.

Donald, P. A., W. T. Stamps, and M. J. Linit. 2003. Pine wilt disease. The Plant Health Instructor. http://dx.doi.org/10. 1094/PHI-I-2003-0130-01

Dowd, F. P. 1992. Insect fungal symbionts: a promising source of detoxifying enzymes. Journal of Industrial Microbiology 9:149-161.

Dybdahl, M. F., and L. S. Kanke. 2005. Adaptation and phenotypic plasticity in the success of a clonal invader. Ecology 86:1592-1601.

Ebeling, S. K., E. Welk, H. Auge, and H. Bruelheide. 2008. Predicting the spread of an invasive plant: combining experiments and ecological niche model. Ecography 31:709719.

EPPO/OEPP. 2009. Diagnostic protocols for regulated pests: Bursaphelenchus xylophilus. Bulletin OEPP/EPPO Bulletin 39:344-353.

Guimarães, P. R., Jr, P. Jordano, and J. N. Thompson. 2011. Evolution and coevolution in mutualistic networks. Ecology Letters 14:877-885.

Himler, A. G., et al. 2011. Rapid spread of a bacterial symbiont in an invasive whitefly is driven by fitness benefits and female bias. Science 332:254-256.

Hulme, P. E. 2008. Contrasting alien and native plant speciesarea relationships: the importance of spatial grain and extent. Global Ecology and Biogeography 17:641-647.

Hyun, M. W., J. H. Kim, D. Y. Suh, S. K. Lee, and S. H. Kim. 2007. Fungi isolated from pinewood nematode, its vector Japanese pine sawyer, and the nematode-infected Japanese black pine wood in Korea. Mycobiology 35:159-161. 
Jiu, M., X. P. Zhou, L. Tong, J. Xu, X. Yang, F. H. Wan, and S. S. Liu. 2007. Vector-virus mutualism accelerates population increase of an invasive whitefly. PLoS ONE 2(1):e182. http://dx.doi.org/10.1371/journal.pone.0000182

Johannesen, J., B. Lux, K. Michel, A. Seitz, and M. Maixner. 2008. Invasion biology and host specificity of the grapevine yellows disease vector Hyalestes obsoletus in Europe. Entomologia Experimentalis et Applicata 126:217-227.

Kanzaki, N., and K. Futai. 2001. Life history of Bursaphelenchus conicaudatus (Nematoda: Aphelenchoididae) in relation to the yellow-spotted longicorn beetle, Psacothea hilaris (Coleoptera: Cerambycidae). Nematology 3:473-479.

Kobayashi, T., K. Sasaki, and Y. Mamiya. 1974. Fungi associated with Bursaphelenchus lignicolus, the pinewood nematode. Journal of Japanese Forest Society 56:136-145.

Linit, M. J. 1988. Nematode-vector relationships in the pine wilt disease system. Journal of Nematology 20:227-235.

Lu, M., M. J. Wingfield, N. E. Gillette, S. R. Mori, and J. H. Sun. 2010. Complex interactions among host pines and fungi vectored by an invasive bark beetle. New Phytologist 187 : 859-866.

Lu, M., M. J. Wingfield, N. E. Gillette, and J. H. Sun. 2011. Do novel genotypes drive the success of an invasive bark beetlefungus complex? Implications for potential reinvasion. Ecology 92:2013-2019.

Lu, M., X. D. Zhou, Z. W. De Beer, M. J. Wingfield, and J. H. Sun. 2009. Ophiostomatoid fungi associated with the invasive pine-infesting bark beetle, Dendroctonus valens, in China. Fungal Diversity 38:133-145.

Maehara, N. 2008. Interactions of pinewood nematodes, woodinhabiting fungi, and vector beetles. Pages 286-298 in B. G. Zhao, J. R. Sutherland, and T. Yuko, editors. Pine wilt disease. Springer, Tokyo, Japan.

Maehara, N., and K. Futai. 2000. Population changes of the pinewood nematode, Bursaphelenchus xylophilus (Nematoda: Aphelenchoididae), on fungi growing in pine-branch segments. Applied Entomology and Zoology 35:413-417.

Mamiya, Y. 1983. Pathology of pine wilt disease caused by Bursaphelenchus xylophilus. Annual Review of Phytopathology 21:201-220.

McLeod, G., R. Gries, S. T. Reuss, J. E. Rahe, R. McIntosh, W. A. König, and G. Gries. 2005. The pathogen causing Dutch elm disease makes host trees attract insect vectors. Proceedings of the Royal Society B 272:2499-2503.

Melbourne, B. A., and A. Hastings. 2009. Highly variable spread rates in replicated biological invasions: fundamental limits to predictability. Science 325:1536-1539.

Moloney, K., C. Holzapfel, K. Tielbörger, F. Jeltsch, and F. Schurr. 2009. Rethinking the common garden in invasion research. Perspectives in Plant Ecology, Evolution and Systematics 11:311-320.

Niu, H. T., L. L. Zhao, M. Lu, S. Zhang, and J. H. Sun. 2012. The ratio and concentration of two monoterpenes mediate fecundity of the pinewood nematode and growth of its associated fungi. PLoS ONE 7(2):e31716. http://dx.doi.org/ 10.1371/journal.pone.0031716
Pan, H. Y., J. R. Ye, and X. Q. Wu. 2009. Spatial distribution patterns of pine wilt disease in China. Acta Ecologica Sinica 29:4325-4331.

Roques, A. 2012. Biological invasion. Integrative Zoology 7: 227.

Shah, M. A., Z. A. Reshi, and I. Rashid. 2012. Synergistic effect of herbivory and mycorrhizal interactions on plant invasiveness. African Journal of Microbiology Research 6:41074112.

Six, D. L., M. Poulsen, A. K. Hansen, M. J. Wingfield, J. Roux, P. Eggleton, B. Slippers, and T. D. Paine. 2011. Anthropogenic effects on interaction outcomes: examples from insectmicrobial symbioses in forest and savanna ecosystems. Symbiosis 53:101-121.

SPSS. 2002. SPSS 11.0 for Windows. SPSS, Chicago, Illinois, USA.

State Forestry Administration of the People's Republic of China. 2011. Announcement of State Forestry Administration of the People's Republic of China, No. 3, 2011.

Sun, J., M. Lu, N. E. Gillette, and M. J. Wingfield. 2013. Red turpentine beetle: innocuous native becomes invasive tree killer in China. Annual Review of Entomology 58:293-311.

Tamura, K., J. Dudley, M. Nei, and S. Kumar. 2007. MEGA4: Molecular Evolutionary Genetics Analysis (MEGA) software version 4.0. Molecular Biology and Evolution 24:1596-1599.

Tennessen, J., and C. Thummel. 2011. Coordinating growth and maturation-insights from Drosophila. Current Biology 21:R750-R757.

Togashi, K., and S. Jikumaru. 2007. Evolutionary change in a pine wilt system following the invasion of Japan by the pinewood nematode, Bursaphelenchus xylophilus. Ecological Research 22:862-868.

von Broembsen, S. L. 1989. Invasions of natural ecosystems by plant pathogens. Pages 77-83 in J. A. Drake, H. A. Mooney, F. Castri, R. H. Groves, F. J. Kruger, M. Rejmanek, and M. Williamson, editors. Biological invasion: a global perspective. John Wiley and Sons, Chichester, UK.

Wang, B., C. Salcedo, M. Lu, and J. H. Sun. 2012. Mutual interactions between an invasive bark beetle and its associated fungi. Bulletin of Entomological Research 102: $71-77$.

Wingfield, M. J. 1987. Fungi associated with the pinewood nematode, Bursaphelenchus xylophilus, and cerambycid beetles in Wisconsin. Mycologia 79:325-328.

Wingfield, M. J., R. A. Blanchette, and T. H. Nicholls. 1984. Is the pinewood nematode an important pathogen in the United States? Journal of Forestry 82:232-235.

Yang, B. J., H. Y. Pan, J. Tang, Y. Y. Wang, L. F. Wang, and Q. Wang. 2003. Bursaphelenchus xylophilus. Chinese Forestry Press, Beijing, China.

Yu, M. J., X. H. Xu, and P. Ding. 2011. Economic loss versus ecological gain: the outbreaks of invaded pinewood nematode in China. Biological Invasions 13:1283-1290.

Zhao, L. L., W. Wei, L. Kang, and J. H. Sun. 2007. Chemotaxis of the pinewood nematode, Bursaphelenchus xylophilus, to volatiles associated with host pine, Pinus massoniana, and its vector Monochamus alternatus. Journal of Chemical Ecology $33: 1207-1216$.

\section{Supplemental Material}

\section{Appendix}

Tables and figures showing the sampling locations, morphological groups of ophiostomatoid isolates, and models of the number of infested trees and frequency of Sporothrix sp. 1 isolates in the field (Ecological Archives E094-260-A1). 